\title{
Independent impairment of osteoblast and osteoclast differentiation in klotho mouse exhibiting low-turnover osteopenia
}

\author{
Hiroshi Kawaguchi, ${ }^{1}$ Noriyo Manabe,${ }^{1}$ Chisato Miyaura, ${ }^{2}$ Hirotaka Chikuda,${ }^{1,3}$ \\ Kozo Nakamura, ${ }^{1}$ and Makoto Kuro-o ${ }^{3}$ \\ ${ }^{1}$ Department of Orthopaedic Surgery, Faculty of Medicine, University of Tokyo, Tokyo 113-8655, Japan \\ ${ }^{2}$ Department of Biochemistry, Tokyo University of Pharmacy and Life Science, Tokyo 192-0392, Japan \\ ${ }^{3}$ Department of Pathology, The University of Texas Southwestern Medical Center at Dallas, \\ Dallas, Texas 75235-9072, USA \\ Address correspondence to: Hiroshi Kawaguchi, Department of Orthopaedic Surgery, Faculty of Medicine, \\ University of Tokyo, 7-3-1 Hongo, Bunkyo, Tokyo 113-8655, Japan. Phone: 81-33815-5411 ext. 33376 or 33375; \\ Fax: 81-33818-4082; E-mail: kawaguchi-ort@h.u-tokyo.ac.jp.
}

Received for publication November 3, 1998, and accepted in revised form June 24, 1999.

\begin{abstract}
We recently identified a new gene, klotho, which is involved in the suppression of multiple aging phenotypes. The mouse homozygous for a disruption of the klotho locus $(k l / k l)$ exhibited multiple pathological conditions resembling human aging. Histomorphometric analysis revealed low-turnover osteopenia in $\mathrm{kl} / \mathrm{kl}$ mice. The decrease in bone formation exceeded that of bone resorption, resulting in a net bone loss. The number of osteoblast progenitors determined by ex vivo bone marrow cultures was reduced in $k l / k l$ mice. In addition, cultured osteoblastic cells derived from $k l / k l$ mice showed lower alkaline phosphatase activity and matrix nodule formation than those from wild-type mice. Osteoclastogenesis in the coculture of marrow cells and osteoblastic cells was decreased only when marrow cells originated from $\mathrm{kl} / \mathrm{kl}$ mice independently of the origin of osteoblastic cells. We also found that the expression of osteoprotegerin, an osteoclastogenesis inhibitor, was significantly upregulated in $\mathrm{kl} / \mathrm{kl}$ mice. We conclude that a defect in the klotho gene expression causes the independent impairment of both osteoblast and osteoclast differentiation, leading to low-turnover osteopenia. Because this state represents a characteristic feature of senile osteoporosis in humans, $\mathrm{kl} / \mathrm{kl}$ mice can be regarded as a useful model for investigating cellular and molecular mechanisms of age-related bone loss.
\end{abstract}

J. Clin. Invest. 104:229-237 (1999).

\section{Introduction}

After the third decade of life, when peak bone mass generally has been achieved, bone is lost with advancing age at a rate that depends on several factors. These factors include the normal aging process as well as the accelerated bone loss associated with menopause in women. The pathophysiology of postmenopausal osteoporosis is characterized by increased bone resorption due to the increased number and activity of osteoclasts. Ovariectomized animals have been used as laboratory models for postmenopausal osteoporosis. The pathophysiology of senile osteoporosis in aged humans observed in both sexes is characterized by a decrease in bone formation that exceeds the decrease in bone resorption and is accompanied by reduced turnover during bone metabolism; however, its cellular and molecular mechanism is poorly understood $(1,2)$.

We recently established a novel mouse model for human aging (3). Insertional mutation of a transgene disrupted a new gene locus, klotho, resulting in premature development of multiple aging phenotypes in the mouse. The klotho gene encodes either membrane or secreted protein through alternative RNA splicing $(4,5)$ that shares sequence similarity with $\beta$-glucosidase enzymes. The mice homozygous for a disruption of the klotho locus ( $\mathrm{kl} / \mathrm{kl}$ or klotho mouse) exhibit osteopenia as a part of the various aging phenotypes, such as a short life span, growth retardation, hypokinesis, infertility, skin atrophy, premature thymic involution, arteriosclerosis, pulmonary emphysema, and ectopic calcification. The klotho gene belongs to a different class of genes from those identified as being responsible for human premature-aging syndromes, such as Werner's syndrome, Cockayne's syndrome, and Bloom's syndrome, which encode DNA helicases and essentially function in the cell nuclei (6-8) The klotho gene may contribute to the aging phenotypes through a completely different molecular pathway from the genes that are involved in the premature-aging syndromes.

The discovery of the klotho mouse provides a unique opportunity to investigate the individual disease mechanisms in the generalized aging background, which is thought to be essential to any study of human aging and age-related diseases. In this report, we investigated the cellular and molecular abnormalities in the bone of klotho mice using in vivo morphologic analyses and ex 

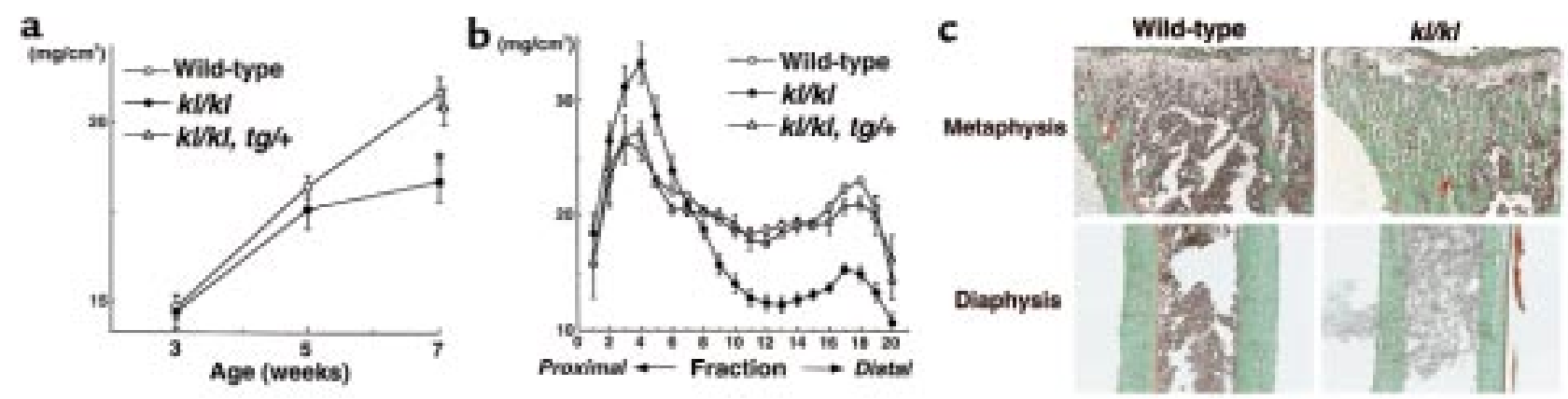

Figure 1

In vivo findings of the tibiae of wild-type, klotho $(\mathrm{kl} / \mathrm{kl})$, and rescued klotho $(\mathrm{kl} / \mathrm{kl}, \mathrm{tg} /+)$ mice. (a) BMD of the whole tibiae at 3,5 , and 7 weeks of age. * Significantly different from wild-type mice $(P<0.01)$. Data are expressed as mean (symbols) \pm SEM (error bars) for 8 bones per group for wild-type and $\mathrm{kl} / \mathrm{kl}$, and 5 bones for $\mathrm{kl} / \mathrm{kl}, \mathrm{tg} /+$. (b) BMD of each of 20 equal longitudinal divisions of tibiae from 7-week-old mice. (c) Histological features of the proximal metaphysis and the diaphysis of the tibiae of 7-week-old wild-type and kl/kl mice. Mineralized bone is stained green and unmineralized osteoid stained red by the Villanueva-Goldner method. Original magnification: $\times 20$. Histomorphometric parameters are shown in Table 1.

vivo cell culture systems. Here we demonstrate the significant similarity between the pathophysiology of osteopenia in klotho mice and that in senile osteoporosis in aged humans. The klotho mice could be a new laboratory animal model for senile osteoporosis and give us a new clue to the understanding of the pathogenetic mechanism of age-related bone loss.

\section{Methods}

Animals. Wild-type and $\mathrm{kl} / \mathrm{kl}$ mice were generated by crossing heterozygous $k l o t h o$ mice $(k l /+)$, because $k l / k l$ mice are infertile. All the aging phenotypes of $\mathrm{kl} / \mathrm{kl}$ mice were rescued by exogenous expression of the klotho gene (3). The "rescued" $\mathrm{kl} / \mathrm{kl}$ mice were obtained by crossing $\mathrm{kl} /+$ mice to transgenic mice overexpressing the membrane form of mouse klotho cDNA under the control of human elongation factor $1 \alpha$ promoter (3). Animals were maintained according to the protocol approved by the Animal Care and Use Committee of the University of Tokyo.

Analysis of bone morphology. Bone mineral density (BMD) of tibiae and femora excised from 3-, 5-, and 7week-old wild-type and $\mathrm{kl} / \mathrm{kl}$ mice, as well as 7-week-old rescued $\mathrm{kl} / \mathrm{kl}$ mice, was measured by single energy $\mathrm{x}$-ray absorptiometry using a bone mineral analyzer (DCS-
600R, Aloka Co., Tokyo, Japan). Bone radiographs of the excised tibiae and femora from 7 -week-old wildtype, $\mathrm{kl} / \mathrm{kl}$, and rescued $\mathrm{kl} / \mathrm{kl}$ mice were taken with a soft $\mathrm{x}$-ray apparatus (Type SRO-M50; Sofron, Tokyo, Japan). Computed tomography (CT) scans at the metaphysis and the diaphysis of femora were taken using a composite $x$-ray analyzing system (NS-ELEX, Tokyo, Japan).

For Villanueva-Goldner staining, tibiae were excised from 7 -week-old wild-type and $k l / k l$ mice, fixed with $100 \%$ ethanol, embedded in methyl methacrylate, and sectioned in $6-\mu \mathrm{m}$ slices. For double labeling, mice were injected subcutaneously with $8 \mathrm{mg} / \mathrm{kg}$ body weight (BW) of calcein at 10 days and 3 days before sacrifice. The specimens were subjected to histomorphometric analyses using a microscope with a video camera connected to an image analysis system. Parameters for the trabecular bone were measured in an area $1.2 \mathrm{~mm}$ long from $0.1 \mathrm{~mm}$ below the growth plate at the proximal metaphysis of the tibiae. Parameters for the cortex bone were measured at the midpoint of the tibiae.

Assay of serum and urine parameters. Serum calcium and phosphorus concentrations were determined by the orthocresolfthalein complexone method using Wako kit 272-21801 (Wako Pure Chemicals Industries, Osaka,

Table 1

Histomorphometry of trabecular and cortex bones of tibiae

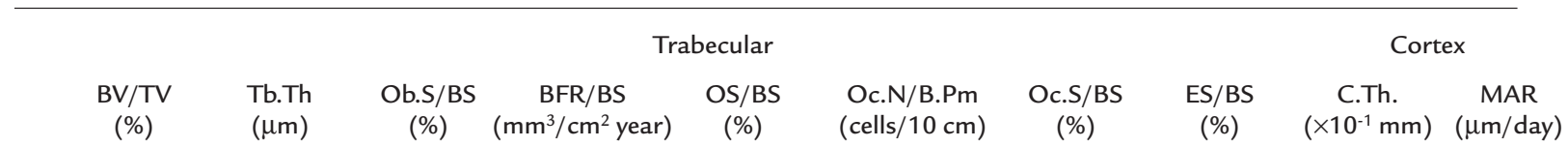

Wild-type $4.34 \pm 0.21 \quad 29.46 \pm 2.2413 .21 \pm 1.6913 .24 \pm 1.06 \quad 27.31 \pm 2.14 \quad 168.16 \pm 19.07 \quad 4.68 \pm 0.7914 .90 \pm 1.73 \quad 2.66 \pm 0.19 \quad 1.76 \pm 0.29$

$\mathrm{kl} / \mathrm{kl} \quad 8.97 \pm 1.22^{\mathrm{A}} 38.41 \pm 4.331 .16 \pm 0.32^{\mathrm{A}} 2.30 \pm 1.49^{\mathrm{A}} \quad 2.58 \pm 0.45^{\mathrm{A}} \quad 62.49 \pm 11.67^{\mathrm{A}} 1.88 \pm 0.01^{\mathrm{A}} 6.52 \pm 0.72^{\mathrm{A}} 1.57 \pm 0.14^{\mathrm{A}} \quad \mathrm{ND}$

Parameters for the trabecular bone were measured in an area $1.2 \mathrm{~mm}$ in length from $0.1 \mathrm{~mm}$ below the growth plate at the proximal metaphysis of the tibiae in Villanueva-Goldner and calcein double labeled sections. Parameters for the cortex bone were measured at the midpoint of the tibiae. Data expressed as means and standard errors for 7 bones per group for wild-type and $k l / k l$. ASignificantly different from wild-type mouse; $P<0.01$. ND, calcein double labels were not detected. BV/TV, trabecular bone volume expressed as a percentage of total tissue volume; Tb. Th, thickness of trabecular bone; Ob.S/BS, percentage of bone surface covered by cuboidal osteoblasts; BFR/BS, bone formation rate expressed by MAR (mineral apposition rate) X percentage of bone surface exhibiting double labels plus one half single labels; OS/BS, percentage of osteoid surface; Oc.N/B.Pm, number of mature osteoclasts in $10 \mathrm{~cm}$ of bone perimeter; Oc.S/BS, percentage of bone surface covered by mature osteoclasts; ES/BS, percentage of eroded surface; C.Th, cortex thickness; MAR, mineral apposition rate. 
Japan) and by the p-methylaminophenol reduction method using Wako kit 270-49801, respectively. For the assay of $1 \alpha, 25(\mathrm{OH})_{2} \mathrm{D}_{3}$ level, $0.3-0.5 \mathrm{~mL}$ of serum from several mice was pooled. Vitamin D metabolites were extracted by the dropwise addition of acetonitrile. The extract was then applied directly to a $\mathrm{C} 18 / \mathrm{OH}$ cartridge (Analytichem International, Harbor City, California, USA) to separate the fraction containing $1 \alpha, 25(\mathrm{OH})_{2} \mathrm{D}_{3}$. The fraction was applied to a HPLC system (Gibson Inc., Middleton, Wisconsin, USA) to obtain a genuine separated residue. Urinary cAMP was determined by radioimmunoassay using a Yamasa kit (Yamasa Shoyu Co. Ltd., Chiba, Japan). Creatinine concentration was determined by the Jaffe method using Wako kit 2771050. Serum osteoprotegerin (OPG) concentration was kindly measured by E. Tsuda at Snow Brand Milk Products Co. Ltd. (Tochigi, Japan) with ELISA using antibodies against human OPG that were ascertained to cross-react with mouse OPG (9).

Ex vivo bone marrow cell cultures. Marrow cells were collected from tibiae and femora of 4- or 7-week-old wild-type or $k l / k l$ mice (each $n=5$ ) by flushing with $\alpha$ modified MEM ( $\alpha$-MEM; GIBCO BRL, Grand Island, New York, USA) containing 10\% FBS using a syringe with a 25-gauge needle. Cells of each group were pooled together and analyzed. The numbers of fibroblast colony-forming units (F-CFU) and osteoblast $\mathrm{CFU}$ (OB-CFU) were determined according to the methods reported previously (10). For F-CFU, $10^{6}$ nucleated bone marrow cells isolated from wild-type and $k l / k l$ mice were inoculated in a well of a 6 -multiwell plate (Corning-Costar, New York, New York, USA), and cultured in $2 \mathrm{~mL} /$ well $\alpha$-MEM containing $10 \% \mathrm{FBS}, 50 \mu \mathrm{g} / \mathrm{mL}$ ascorbic acid, and $10 \mathrm{mM} \beta$-glycerophosphate. After 10 days of culture with half of the medium changed every 3 days, the cells were fixed with $10 \%$ neutral buffered formalin and stained for alkaline phosphatase (ALP) by enzyme histochemical procedure using naphthol AS-MX phosphate (Sigma Chemical Co., St. Louis, Missouri, USA) as substrate and Fast BB salt (Sigma Chemical Co.) as coupler. FCFU was detected by the formation of a colony of more than 50 adherent fibroblastic cells. For OB$\mathrm{CFU}, 10^{6}$ nucleated bone marrow cells were inoculated in a well of a 6-multiwell plate and cultured in the same medium for 28 days, fixed in $50 \%$ ethanol and $18 \%$ formaldehyde, and stained for calcium deposits

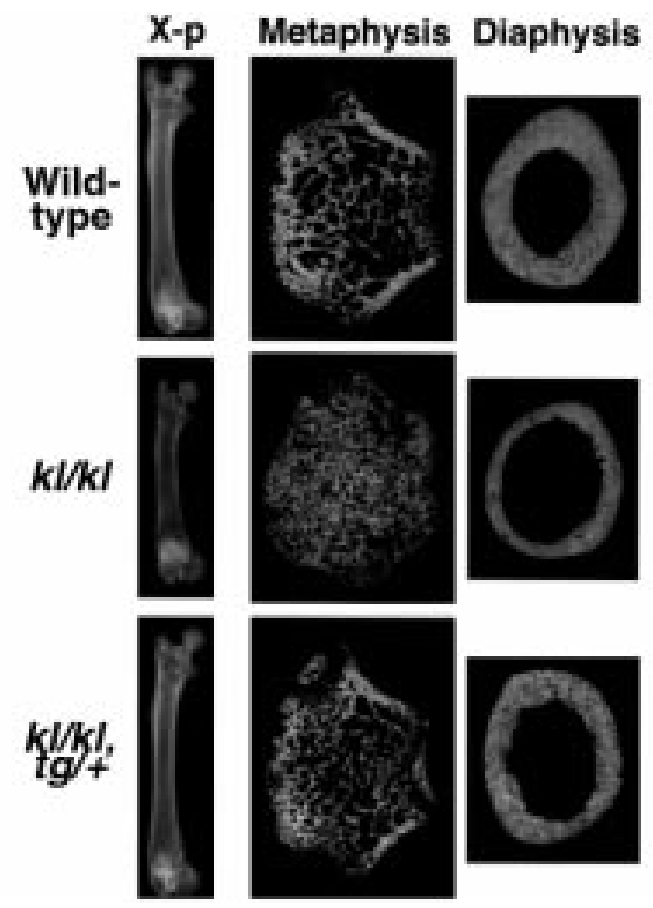

Figure 2

Plain x-rays and CT images of the femora of 7-week-old wild-type, $\mathrm{kl} / \mathrm{kl}$, and $\mathrm{kl} / \mathrm{kl}, \mathrm{tg} /+$ mice. The length of the femora, as well as the tibiae, of $k l / k l$ mice was $20-30 \%$ shorter than those of wild-type mice, reflecting growth retardation in $\mathrm{kl} / \mathrm{kl}$ mice. CT was taken at $0.2 \mathrm{~mm}$ above the growth plate at the distal metaphysis and at the midportion at the diaphysis.

using $2 \%$ alizarin red or Von Kossa's method. The colonies exhibiting a nodule of mineralized matrix were counted as OB-CFU.

Granulocyte and macrophage CFU (GM-CFU) were determined according to the method reported previously (11). Briefly, $10^{6}$ nucleated bone marrow cells from 7 -week-old wild-type or $\mathrm{kl} / \mathrm{kl}$ mice were inoculated in a well of a 6-multiwell plate and cultured in 2 $\mathrm{mL} /$ well of $\alpha$-MEM containing $10 \%$ FBS, $0.88 \%$ methylcellulose, 500 U GM-CSF (Genzyme Pharmaceuticals, Cambridge, Massachusetts, USA), and $100 \mu \mathrm{M} 2$-mercaptoethanol. After 8 days of culture, cells were fixed and stained for nonspecific esterase and naphthol ASD chloroacetate esterase using commercially available kits (Sigma Chemical Co.). Colonies of more than 50 cells were counted as GM-CFU.

Table 2

F-CFU and OB-CFU in cultured bone marrow cells from wild-type and $k l / k l$ mice at 4 and 7 weeks of age

\begin{tabular}{|c|c|c|c|c|}
\hline & \multicolumn{2}{|c|}{4 weeks old } & \multicolumn{2}{|c|}{7 weeks old } \\
\hline & Wild-type & $\mathrm{kl} / \mathrm{kl}$ & Wild-type & $\mathrm{kl} / \mathrm{kl}$ \\
\hline F-CFU & $34.43 \pm 4.31$ & $28.16 \pm 3.65$ & $28.54 \pm 2.91$ & $24.81 \pm 1.69$ \\
\hline OB-CFU & $12.97 \pm 1.81$ & $4.56 \pm 1.01^{\mathrm{A}}$ & $15.95 \pm 1.72$ & $5.26 \pm 1.50^{\mathrm{A}}$ \\
\hline
\end{tabular}

Marrow cells were collected from tibiae and femora of 4 or 7 week-old wild-type or $k l / k l$ mice (each $n=5$ ) and pooled together in each group. The number of colonies per well formed ex vivo cultures of marrow cells $\left(10^{6}\right.$ cells per well) were counted after the culture periods as mentioned in Methods. Data are expressed as the mean \pm SEM for 6 wells per group. ${ }^{A} P<0.01$ vs. wild-type. F-CFU, fibroblast colony-forming units; OB-CFU, osteoblast colony-forming units. 

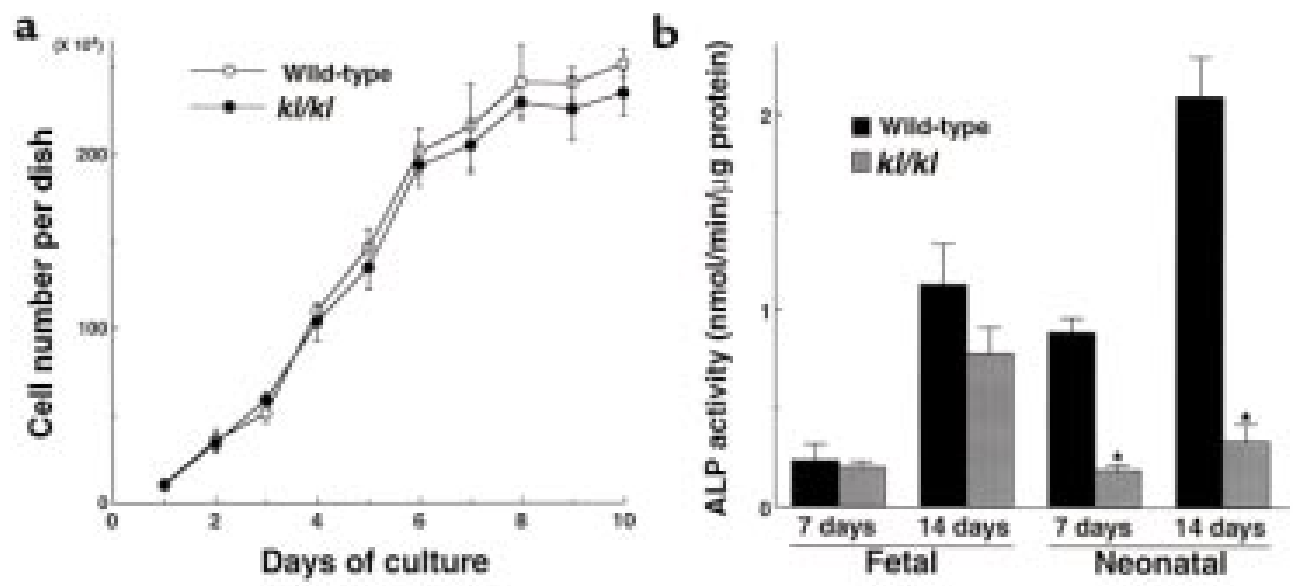

c

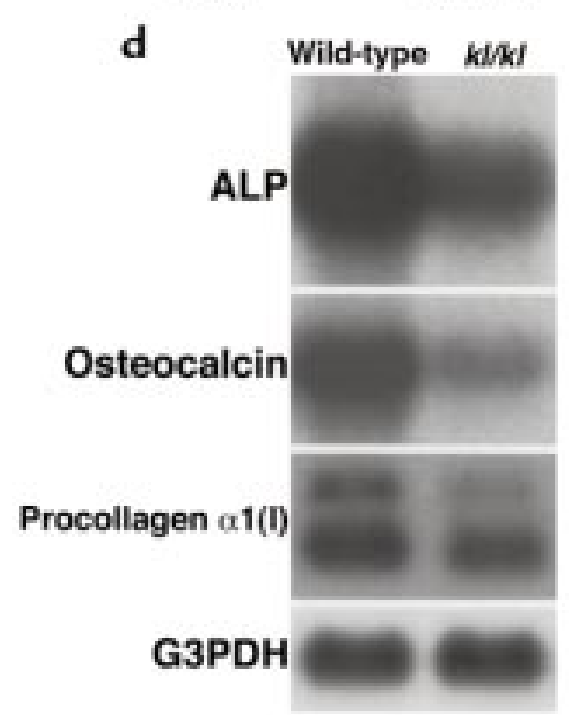

\section{Figure 3}

Cultures of osteoblastic cells from wild-type and $\mathrm{kl} / \mathrm{kl}$ mice. (a) Growth curve of cultured osteoblastic cells from neonatal mouse calvariae during 10 days of culture. Data are expressed as mean (symbols) \pm SEM (error bars) of pooled data from 3 independent experiments (6 dishes per group). (b) ALP activity of cultured osteoblastic cells from fetal ( 17 days after coitus) and neonatal (5-6 days old) wild-type or kl/kl mouse calvariae at 7 and 14 days of culture. Data are expressed as mean (bars) \pm SEM (error bars) for 8 wells per group. ${ }^{*}$ Significantly different from wild-type mice $(P<0.01)$. (c) Representative pictures of alizarin red-stained matrix nodule formation by cultured osteoblastic cells from fetal and neonatal wild-type or $\mathrm{kl} / \mathrm{kl}$ mouse calvariae at 21 days of culture. Areas of positively stained matrix nodule were $35.3 \pm$ $4.1 \mathrm{~mm}^{2}$ for fetal wild-type, $31.1 \pm 2.5 \mathrm{~mm}^{2}$ for fetal $\mathrm{kl} / \mathrm{kl}, 53.4 \pm 6.3 \mathrm{~mm}^{2}$ for neonatal wild-type, and $8.7 \pm 3.1 \mathrm{~mm}^{2}$ for neonatal $\mathrm{kl} / \mathrm{kl}$ (all mean \pm SEM for 6 wells per group). Significant difference $(P<0.01)$ between wild-type and $\mathrm{kl} / \mathrm{k} /$ was seen in cultured osteoblastic cells from neonatal mice, but not in those from fetal mice. (d) Steady-state mRNA levels of ALP, osteocalcin, and procollagen $\alpha 1$ (I) in cultured osteoblastic cells from neonatal wild-type or $\mathrm{kl} / \mathrm{kl}$ mouse calvariae at 7 days of culture, as determined by Northern blot analysis.

To determine osteoclastogenesis, $10^{6}$ nucleated bone marrow cells from 7-week-old wild-type or $k l / k l$ mice were inoculated in a well of a 24-multiwell plate and cultured in $0.5 \mathrm{~mL} /$ well $\alpha$-MEM containing $10 \% \mathrm{FBS}$, $50 \mu \mathrm{g} / \mathrm{mL}$ ascorbic acid, and $10^{-8} \mathrm{M} 1 \alpha, 25(\mathrm{OH})_{2} \mathrm{D}_{3}$ for 8 days. Cells were fixed with $3.7 \%$ (vol/vol) formaldehyde in PBS and ethanol-acetone (50:50 vol/vol), and stained at $\mathrm{pH} 5.0$ in the presence of $\mathrm{L}(+)$-tartaric acid using naphthol AS-MX phosphate in $N, N$-dimethyl formamide as the substrate. Tartrate-resistant acid phosphatase-positive (TRAP-positive) multinucleated with 3 or more nuclei were counted as osteoclastic cells.

Ex vivo osteoblastic cell cultures. Osteoblastic cells were isolated from 6 to 8 calvariae of neonatal (5-6 days old) or fetal ( 17 days after coitus) wild-type and $k l / k l$ mice.
Calvariae were digested for 10 minutes at $37^{\circ} \mathrm{C} 5$ times in an enzyme solution containing $0.1 \%$ collagenase and $0.2 \%$ dispase in $\alpha$-MEM. Cells isolated by the last 4 digestions were combined as an osteoblastic cell population and cultured in $\alpha$-MEM containing 10\% FBS and $50 \mu \mathrm{g} / \mathrm{mL}$ ascorbic acid. For the growth-curve experiment, primary osteoblastic cells from neonatal mice were inoculated at a density of $10^{5}$ cells per dish in $6-\mathrm{cm}$ dishes. The number of cells per dish was counted every day for 10 days. For ALP activity measurement, primary osteoblastic cells from neonatal or fetal mice were inoculated at a density of $5 \times 10^{4}$ cells per well in a 24-multiwell plate and cultured in the same medium. At 7 and 14 days of culture, the cells were washed with PBS and sonicated in $10 \mathrm{mM}$ Tris- $\mathrm{HCl}$ buffer ( $\mathrm{pH}$ 8.0) 
containing $1 \mathrm{mM} \mathrm{MgCl}$ and $0.5 \%$ Triton X-100. ALP activity in the lysate was measured by the hydrolysis of p-nitrophenyl phosphate to $\mathrm{p}$-nitrophenol. The protein content was determined using BCA protein assay reagent (Pierce Chemical Co., Rockford, Illinois, USA). For matrix nodule formation, primary osteoblastic cells from neonatal or fetal mice were inoculated at a density of $10^{5}$ cells per well in a 6-multiwell plate and cultured in $\alpha$-MEM containing $10 \%$ FBS, $50 \mu \mathrm{g} / \mathrm{mL}$ ascorbic acid, $10 \mathrm{nM}$ dexamethasone, and $10 \mathrm{mM}$ $\beta$-glycerophosphate for 21 days. Matrix nodules were identified morphologically by alizarin red staining, and total area of positively stained matrix was estimated using an image analyzer.

Coculture of osteoblastic cells and hemopoietic cells. TRAPpositive multinucleated osteoclastic cells were generated from the hemopoietic cells derived from either wildtype or $\mathrm{kl} / \mathrm{kl}$ mice by coculturing them with the osteoblastic cells derived from either wild-type or $\mathrm{kl} / \mathrm{kl}$ mice. As a source of hemopoietic cells including osteoclast progenitors, we used bone marrow cells and spleen cells that were collected from 7-week-old mice. Osteoblastic cells $\left(5 \times 10^{4}\right.$ cells per well $)$ isolated from neonatal mice as described above were cocultured with bone marrow cells $\left(5 \times 10^{5}\right.$ cells per well $)$ or spleen cells $\left(10^{6}\right.$ cells per well) in a $24-$ multiwell plate in $0.5 \mathrm{~mL} /$ well of $\alpha$-MEM containing $10 \%$ FBS and $10^{-8} \mathrm{M}$ $1 \alpha, 25(\mathrm{OH})_{2} \mathrm{D}_{3}$ for 8 days. For the determination of osteoclastogenesis, cells were stained for TRAP as described above. To determine the survival of osteoclasts, osteoclastic cells formed in 8 days of the coculture were collected by removing other cells with $0.001 \%$ pronase $\mathrm{E}$ and $0.02 \%$ EDTA and cultured in $\alpha$-MEM containing $10 \%$ FBS for an additional 48 hours. At 4, 8, 12, 18, 24, 36 , and 48 hours, the number of TRAP-positive and trypan blue-negative multinucleated cells was counted, and the half-life was calculated from the survival curve. For the determination of bone resorption activity, osteoclastic cells were formed by the coculture for 8 days on $0.24 \%$ collagen gel coated on a 24 -multiwell plate. After cells were digested with $0.2 \%$ collagenase solution, a 1:50 aliquot, including osteoclastic cells, was seeded on a dentine slice and further cultured in $\alpha$-MEM containing $10 \%$ FBS for 48 hours. To compare the resorption activity of the osteoclastic cells of wild-type mice with that of $\mathrm{kl} / \mathrm{kl}$ mice, the same number of TRAP-positive multinucleated cells was inoculated on a dentine slice, and total area of pits stained with $0.5 \%$ toluidine blue was evaluated using an image analyzer.

Immunofluorescence analysis of bone marrow cells. The bone marrow cells collected from tibiae and femora of 7-week-old mice were centrifuged and resuspended in ammonium chloride-Tris buffer to lyse red blood cells, then incubated for 30 minutes on ice with FITC-labeled F4/80 (Serotec Ltd., Oxford, United Kingdom). Stained cells were analyzed by a flow cytometer (Cyto ACE-150; Japan Spectroscopic Co., Tokyo, Japan).

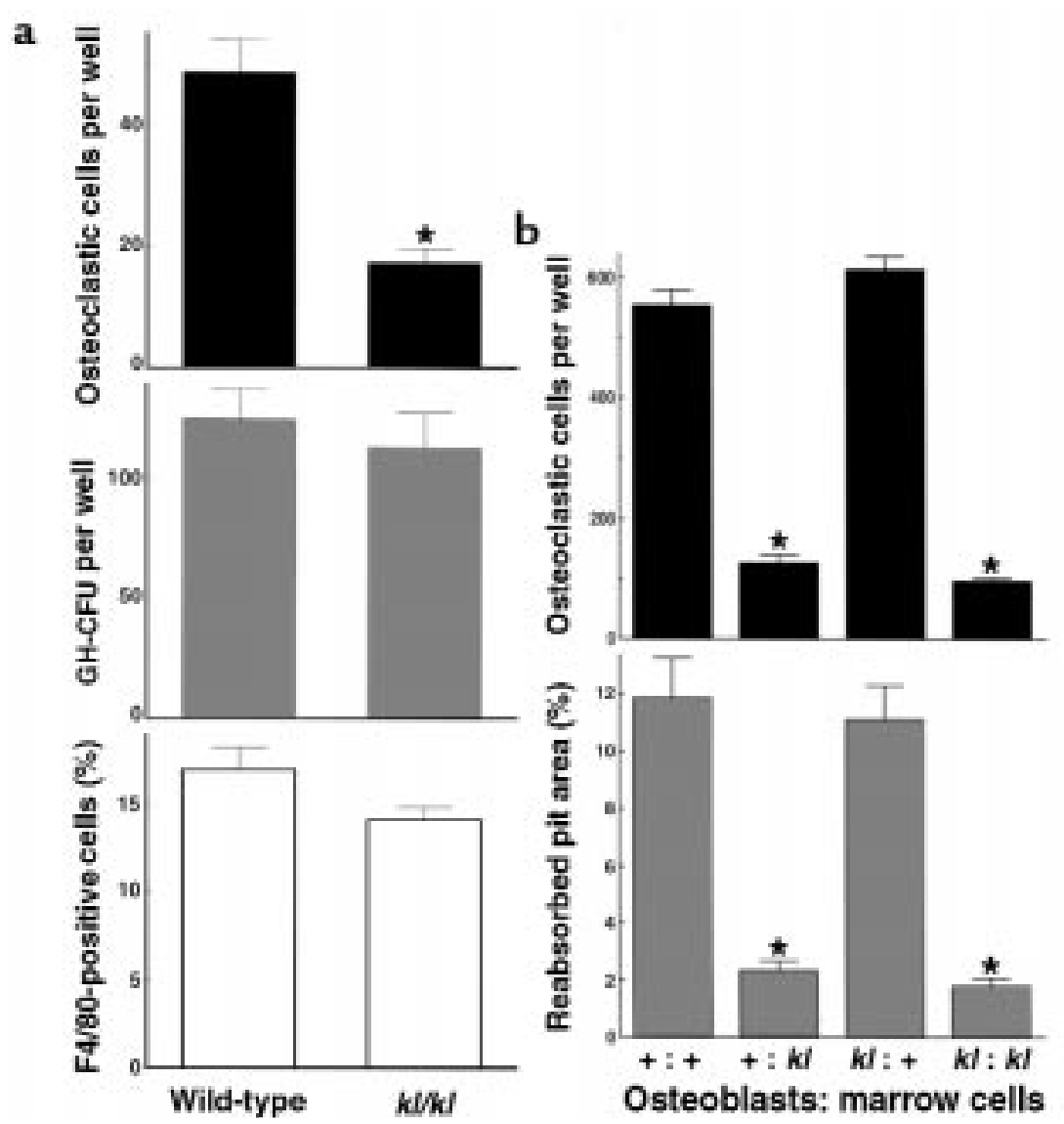

\section{Figure 4}

Osteoclastogenesis in ex vivo bone marrow cell cultures (a) and in the coculture of osteoblastic cells and bone marrow cells (b) from wild-type mice and $\mathrm{kl} / \mathrm{kl}$ mice. (a) The number of TRAPpositive osteoclastic cells (top) and GM-CFU (middle) at 8 days of culture of bone marrow cells from 7-week-old wild-type or $\mathrm{kl} / \mathrm{kl}$ mice. Data are expressed as mean (bars) \pm SEM (error bars) for 8 and 6 wells per group, respectively. * Significantly different from wild-type cells $(P<0.01)$. The percentage of $\mathrm{F} 4 / 80$-positive monocyte macrophages in bone marrow cells was measured by flow cytometric analysis using FITC-labeled F4/80 (bottom). (b) The number of TRAP-positive osteoclastic cells formed at 8 days of coculture (top) and the pit area resorbed by osteoclastic cells by an additional 48 hours of culture on a dentine slice (bottom; percent of whole dentine). The origins ( + for wild-type mice and $\mathrm{kl}$ for $\mathrm{kl} / \mathrm{kl}$ mice) of the cells are indicated as "Osteoblasts: marrow cells." Data are expressed as mean (bars) \pm SEM (error bars) for 8 wells per group. *Significantly different from wild-type cultures $(P<0.01)$. 
Northern blot analysis and RT-PCR. For Northern blot analysis, primary osteoblastic cells from neonatal mouse calvariae were inoculated at a density of $10^{6} \mathrm{cells}$ per dish in a $10-\mathrm{cm}$ dish and cultured in $\alpha$-MEM containing $10 \% \mathrm{FBS}$ and $50 \mu \mathrm{g} / \mathrm{mL}$ ascorbic acid for 7 days. Total RNA was extracted using an ISOGEN kit (Wako Pure Chemicals Industries). Twenty micrograms of total RNA was electrophoresed in $1.2 \%$ agaroseformaldehyde gels and transferred onto nylon membrane filters (Hybond-N; Amersham International, Little Chalfont, United Kingdom). The membranes were hybridized for 2 hours at $6^{\circ} \mathrm{C}$ with cDNA probes for mouse ALP, osteocalcin, procollagen $\alpha 1$ (I), and G3PDH, which were labeled using a multirandom primer oligonucleotide labeling kit (Boehringer Mannheim GmbH, Mannheim, Germany), and [32 P]dCTP (Amersham International), according to the manufacturer's protocol.

To investigate in vivo levels of mRNA, semiquantitative RT-PCR was performed within the exponential phase of the amplification. Total RNA $(1 \mu \mathrm{g})$ extracted from calvariae and tibiae of 7 -week-old mice was reverse transcribed using SuperScript reverse transcriptase (Takara Shuzo Co., Shiga, Japan) with random hexamer (Takara Shuzo Co.), and $5 \%$ of the reaction mixture was amplified with LA-Taq DNA polymerase (Takara Shuzo Co.) using specific primer pairs: 5'-CCTGGTCGACCATTTCAG-3' and $5^{\prime}$-AGCACAAAGTCGACAGACTTCTGGC-3' for klotho; 5'-CCGCACGACAACCGCACCAT-3' and 5'CGCTCCGGCCCACAAATCTC-3' for Cbfa-1; 5'-GACGGACTGCGGTCTCCTAAAG-3' and $5^{\prime}$-TCTGCAGAGTGAGAAACTCGTCA-3' for BMP-2; 5'-CGCCGTCATTCCG- GATTACAT-3' and 5'-GGCCCAATCTCCACTCCCTT-3' for BMP-4; 5'-TAGCACCAGAGGATACCTTGC- $3^{\prime}$ and $5^{\prime}$-AATGCTTCATCC GTTCCAAA- $3^{\prime}$ for BMPR-IA; 5'-CAGAATCAAGAACGGCTATG-3' and 5'-TTGTTTACGGTCTCCTGTCA-3' for BMPR-II; 5' TCAGAAGACAGCACTCACTG-3' and $5^{\prime}$ TCTTCACCAGCTCGGAGCTT-3' for ODF; 5'-CACCAAAAGTGTGGAATAG- $3^{\prime}$ and $5^{\prime}$-GTGGGATGTTTTCAAGTGCT-3' for OPG; 5'-CATGTAGGCCATGAGGTCCACCAC-3' and 5'-TGAAGGTCGGTGTGAACGGATTTGGC-3' for G3PDH. Up to 30 cycles of amplification were performed with a Perkin-Elmer PCR Thermal Cycler (PE-9600; Perkin-Elmer Corp., Norwalk, Connecticut, USA ) at $94^{\circ} \mathrm{C}$ for 30 seconds, at $51-65^{\circ} \mathrm{C}$ for 60 seconds, and at $72^{\circ} \mathrm{C}$ for 90 seconds.

Statistical analysis. Means of groups were compared by ANOVA, and significance of differences was determined by post hoc testing using the Bonferroni method.

\section{Results}

Radiological and histological analyses of the bone phenotypes. BMD of whole tibiae was decreased by $12.0 \%$ in $\mathrm{kl} / \mathrm{kl}$ mice at 7 weeks of age compared with wild-type mice (Figure 1a). To investigate the distribution of BMD, we divided the tibiae longitudinally into 20 equal regions and measured BMD of each fraction (Figure 1b). In $\mathrm{kl} / \mathrm{kl}$ mice, BMD was significantly reduced at the midportion or the diaphysis with a maximal decrease of $35.9 \%$. On the other hand, BMD of the proximal tibiae or the metaphysis was greater in $\mathrm{kl} / \mathrm{kl}$ mice than in wildtype mice, with the maximal increase of $29.1 \%$. These findings were not observed in the genetically rescued $\mathrm{kl} / \mathrm{kl}$ mice $(\mathrm{kl} / \mathrm{kl}, \mathrm{tg} /+)$ generated by crossing klotho mice to the transgenic mice overexpressing the klotho cDNA

Histological examination of the tibiae of 7-week-old wild-type and $k l / k l$ mice (Figure $1 c$ ) revealed that the decrease in BMD at the diaphysis in $\mathrm{kl} / \mathrm{kl}$ mice was caused by the decrease in cortex thickness. It was also indicated that the dense and elongated trabecular bone at the metaphysis was responsible for the increased BMD in this region in these mice. These elongated metaphyseal trabeculae exhibited a lamellar architecture by polarizing microscope (data not shown). Histomorphometric measurements supported these his-
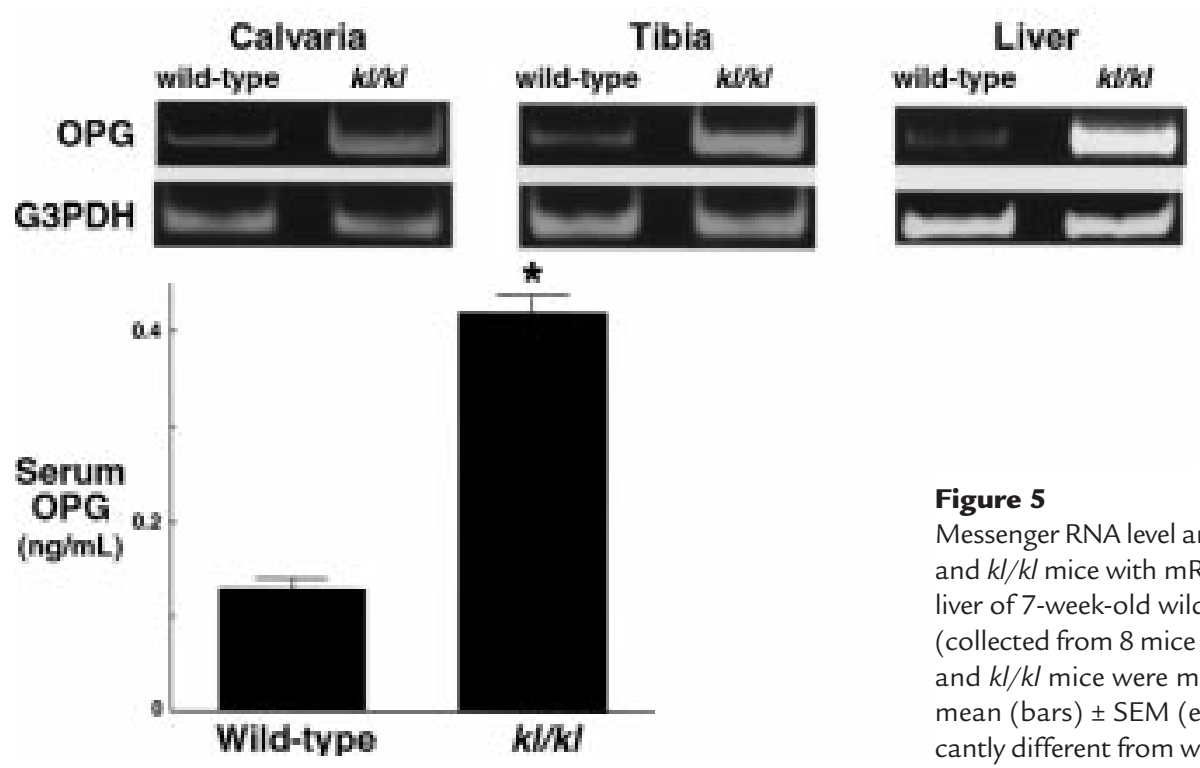

(100 
tological observations (Table 1). Cortex bone thickness was decreased by approximately $40 \%$, whereas trabecular bone volume and thickness were increased by approximately $100 \%$ and $30 \%$, respectively. Histomorphometric parameters for bone formation (osteoblast surface per bone surface [Ob.s/BS] and bone formation rate per surface $[\mathrm{BFR} / \mathrm{BS}])$ in $\mathrm{kl} / \mathrm{kl}$ mice were much lower than those in wild-type mice. In addition, parameters for bone resorption (osteoclast number per bone perimeter [Oc.N/B.Pm], osteoclast surface per bone surface [Oc.S/BS], and eroded surface per bone surface [ES/BS]) were also significantly reduced, indicating a state of low bone turnover in $\mathrm{kl} / \mathrm{kl}$ mice. The decrease in bone formation parameters (80-90\% reduction) exceeded the decrease in bone resorption parameters ( $60 \%$ reduction), which is a characteristic feature of senile osteoporosis in aged humans (1).

Plain x-ray and CT of femora demonstrated similar changes to the tibiae (Figure 2). BMD of the whole femur was decreased by $5.0 \%$ in $\mathrm{kl} / \mathrm{kl}$ mice with the maximal decrease of $25.8 \%$ at the diaphysis, whereas dense trabecular bones were observed at the distal metaphysis, which caused the maximal increase of $23.4 \%$ in BMD in this region. These changes seen in $\mathrm{kl} / \mathrm{kl}$ mice were not detected in rescued $\mathrm{kl} / \mathrm{kl}$ mice. No sex differences were apparent for any of these morphologic findings of tibiae or femora.

Analysis of serum and urine parameters. The serum level of calcium was slightly increased in $\mathrm{kl} / \mathrm{kl}$ mice $(9.47 \pm$ 0.30 vs. $10.64 \pm 1.07 \mathrm{mg} / \mathrm{dL}$ [mean $\pm \mathrm{SD}$ ] for wild-type and $\mathrm{kl} / \mathrm{kl}$ mice, respectively; $n=12$ ). The serum phosphorus was also increased (8.54 \pm 1.34 vs. $15.09 \pm 1.34$ $\mathrm{mg} / \mathrm{dL}$ ) as reported previously (3). The mechanism of the elevated calcium and phosphorus levels remains to be determined; however, renal function seemed unaffected, because creatinine levels were normal in $k l / k l$ mice. No difference was evident between wild-type and $\mathrm{kl} / \mathrm{kl}$ mice in serum $1 \alpha, 25(\mathrm{OH})_{2} \mathrm{D}_{3}$ level (52.1 $\pm 4.6 \mathrm{vs}$. $45.9 \pm 7.9 \mathrm{pg} / \mathrm{mL}[$ mean $\pm \mathrm{SD}]$ for wild-type and $\mathrm{kl} / \mathrm{kl}$ mice, respectively; $n=8$ ) and urinary cAMP/creatinine ratio $(74.47 \pm 7.98$ vs. $68.11 \pm 6.54 \mathrm{nmol} / \mathrm{mgCr})$, which represents parathyroid hormone (PTH) activity. In addition, immunohistochemical study of the parathyroid gland using an anti-PTH antibody provided no evidence for hypertrophy in $\mathrm{kl} / \mathrm{kl}$ mice (data not shown). Other serum data, including total protein, albumin, cholesterol, and triglyceride levels, were normal in these mice. These observations confirmed that the bone phenotypes seen in $\mathrm{kl} / \mathrm{kl}$ mice were not caused by a metabolic disorder such as renal failure or hyperparathyroidism.

Osteoblast progenitors in the bone marrow. Marrow cells from 4- and 7-week-old $\mathrm{kl} / \mathrm{kl}$ mice had approximately 3 -fold fewer OB-CFU than did wild-type mice in ex vivo cultures (Table 2), indicating that the number of osteoblast progenitors was significantly decreased in the bone marrow of $\mathrm{kl} / \mathrm{kl}$ mice. Both 4-week and 7week-old wild-type and $\mathrm{kl} / \mathrm{kl}$ mice, in contrast, had a similar number of F-CFU, although the colonies origi- nated from $\mathrm{kl} / \mathrm{kl}$ mice showed less ALP staining than those from wild-type mice (data not quantified).

Analysis of cultured osteoblastic cells. Cultured primary osteoblastic cells from neonatal (5-6 days old) calvariae showed no significant difference in proliferation between wild-type and $\mathrm{kl} / \mathrm{kl}$ mice (Figure 3a); however, osteoblastic cells from the $\mathrm{kl} / \mathrm{kl}$ mice had lower ALP activity at 7 and 14 days of culture (Figure 3b). Matrix nodule formation by cultured osteoblastic cells was also decreased at 21 days of culture (Figure 3c). Northern blot analysis disclosed the decrease in mRNA levels of ALP, osteocalcin, procollagen $\alpha 1$ (I) in $\mathrm{kl} / \mathrm{kl}$ osteoblastic cells (Figure $3 \mathrm{~d}$ ). These results indicate that not only the number of osteoblast progenitors, but also the function of their progeny (osteoblastic cells), were severely affected in $\mathrm{kl} / \mathrm{kl}$ mice. It should be noted that ALP activity (Figure $3 \mathrm{~b}$ ) and matrix formation (Figure $3 \mathrm{c}$ ) of $\mathrm{kl} / \mathrm{kl}$ mice were almost equivalent to those of wild-type mice when osteoblastic cells were derived from embryos (17 days after coitus).

Osteoclastogenesis. TRAP-positive multinucleated osteoclastic cell formation was impaired in $\mathrm{kl} / \mathrm{kl}$ mice in ex vivo bone marrow cultures (Figure 4a). It is firmly established that osteoclasts differentiate from hemopoietic cells through cell-cell interaction with stromal/osteoblastic cells (12). Hence, impairment of osteoclastogenesis can be caused by an intrinsic defect in osteoclast progenitors to differentiate to mature osteoclasts or a defect in stromal/osteoblastic cells to support osteoclast differentiation or both. To determine the cause of impaired osteoclastogenesis in $\mathrm{kl} / \mathrm{kl}$ mice, we counted the number of osteoclastic cells generated in the coculture of primary osteoblastic cells and bone marrow cells. This number was significantly decreased when bone marrow cells were derived from $\mathrm{kl} / \mathrm{kl}$ mice; however, it was unaffected by whether the osteoblastic cells were derived from wild-type mice or $\mathrm{kl} / \mathrm{kl}$ mice (Figure $4 \mathrm{~b}$, top). This result was reproducible when bone marrow cells were replaced by spleen cells as a source of hemopoietic cells (data not shown). Thus, we concluded that the impairment of osteoclastogenesis in $\mathrm{kl} / \mathrm{kl}$ mice was due to abnormality of the bone marrow cells containing osteoclast progenitors and was not secondary to a defect of osteoblastic cells to support osteoclast differentiation. We found that the number of GM-CFU in ex vivo bone marrow culture and the percentage of F4/80-positive monocyte macrophages in bone marrow cells were identical in wild-type and $\mathrm{kl} / \mathrm{kl}$ mice (Figure $4 \mathrm{a}$ ), suggesting that these cell lineages were not affected in $\mathrm{kl} / \mathrm{kl}$ mice.

Activity and survival of osteoclasts. When osteoclastic cells formed in the coculture system were isolated and further cultured on a dentine slice for 48 hours, the area of resorption pit was parallel to the number of osteoclastic cells formed (Figure $4 b$ ). In addition, the osteoclastic cells originated from $\mathrm{kl} / \mathrm{kl}$ mice formed a resorption pit almost equal in area to that formed by wild-type mice when the same number of cells were seeded (resorption pit area of $\mathrm{kl} / \mathrm{kl}$ osteoclasts was 87.3 
$\pm 10.2 \%$ that of wild-type osteoclasts; mean \pm SEM). Survival curves showed no difference between wild-type and $k l / k l$ osteoclasts (the half-life of osteoclastic cells was 14.4 hours and 12.9 hours, respectively). These results strongly suggest that the decrease in bone resorption in $\mathrm{kl} / \mathrm{kl}$ mice is caused by a defect in osteoclast differentiation from their progenitors, but not by reduced activity or survival of mature osteoclasts.

Analysis of major regulatory factors of bone metabolism. To obtain an insight into molecular mechanisms of the bone cell abnormalities in $\mathrm{kl} / \mathrm{kl}$ mice, we compared expression levels of several genes that regulate bone development and metabolism between wild-type and $\mathrm{kl} / \mathrm{kl}$ mice. We examined expression of Cbfa-1 (13), bone morphogenetic protein-2 (BMP-2), BMP-4, BMP receptor-IA (BMPR-IA), BMPR-II (14), osteoclast differentiation factor (ODF) $(15)$, and OPG $(16,17)$ by semiquantitative RT-PCR using RNA from calvariae and tibiae of 7-week-old wild-type and $k l / k l$ mice. No difference was evident in expression levels of Cbfa-1, BMP-2, BMP-4, BMPR-IA, BMPR-II, and ODF (data not shown). Significant difference was detected in OPG, a secreted factor that inhibits osteoclastogenesis (Figure 5). In $k l / k l$ mice, OPG mRNA level was increased both in bones ( 15 -fold- that of wild-type mice in tibiae by competitive RT-PCR) and in liver, as well as in cultured osteoblastic cells (data not shown). The serum level of OPG protein was about 3-fold higher than that in wild-type mice (Figure 5).

\section{Discussion}

In this study, we analyzed the pathophysiology of osteopenia observed in $\mathrm{kl} / \mathrm{kl}$ mice, a novel laboratory animal model for human aging caused by a mutation of the mouse klotho gene. Osteopenia in $\mathrm{kl} / \mathrm{kl}$ mice was accompanied by reduced turnover during bone metabolism, in which the decrease in bone formation exceeded the decrease in bone resorption. This state closely resembles that of senile osteoporosis in aged humans. We also revealed a defect of differentiation of both osteoblasts and osteoclasts in $\mathrm{kl} / \mathrm{kl}$ mice through ex vivo bone and bone marrow cell cultures. In addition, we found that expression of OPG that suppresses osteoclast differentiation was significantly increased in $\mathrm{kl} / \mathrm{kl}$ mice. We conclude that these cellular and molecular abnormalities caused by a defect of the klotho gene expression underlie the pathophysiology of lowturnover osteopenia in $\mathrm{kl} / \mathrm{kl}$ mice.

Evidence is accumulating to indicate that the pleiotropic klotho gene functions are mediated by humoral factor(s) (3). First, the klotho gene is expressed in specific cells and tissues such as kidney or brain despite the fact that a defect in klotho expression causes systemic aging phenotypes. Many organs affected in $\mathrm{kl} / \mathrm{kl}$ mice, including bone and bone marrow, do not express the klotho gene endogenously (3). Second, exogenous expression of this gene can improve all the aging phenotypes in $k l / k l$ mice even when the exogenous klotho expression is limited to a few organs (3).
Last, parabiosis between wild-type and $k l /+$ mice improves vascular endothelial dysfunction observed in $k l /+$ mice (18). We recently identified a splice variant of the klotho gene that encodes a putative secreted protein $(4,5)$. It is possible that klotho protein (KL protein) itself may function as a circulating humoral factor or "antiaging" hormone. Alternatively, based on the homology to $\beta$-glucosidases, KL protein may function as a $\beta$-glucosidase-like enzyme and modify a factor(s) involved in the humoral signaling pathway. Therefore, it is distinctly possible that KL protein controls bone cell differentiation through a humoral signaling pathway, although the klotho gene is not expressed in the bone or bone marrow (3). Furthermore, the impairment of differentiation seen in neonatal $k l / k l$ mouse osteoblastic cell cultures was not reproducible in fetal $\mathrm{kl} / \mathrm{kl}$ mouse osteoblastic cell cultures (Figure 3, b and c). Because the klotho gene transcript could not be detected during embryonic development up to 16 days after coitus, but could be detected in the kidney of newborn (day 1) wild-type mice (3), in vivo exposure of the osteoblastic cells to the systemic klotho signaling during the perinatal period may be essential to their commitment to normal differentiation.

The bone marrow of $k l / k l$ mice had fewer osteoblast progenitors or OB-CFU than that of wild-type mice, but a normal number of multipotential mesenchymal progenitors or F-CFU. Similarly, the bone marrow of $\mathrm{kl} / \mathrm{kl}$ mice had fewer osteoclast progenitors, but had a normal number of hemopoietic progenitors, GM-CFU, and F4/80-positive cells. These observations indicate that osteoblastogenesis and osteoclastogenesis may be selectively suppressed in the bone marrow of $k l / k l$ mice. It should be noted that ALP activity and matrix formation of osteoblastic cells were impaired in $\mathrm{kl} / \mathrm{kl}$ mice, whereas the resorbing activity and survival of osteoclastic cells were not affected. This may partly explain the greater reduction in bone formation than in bone resorption in $k l / k l$ mice.

OPG is a humoral factor that inhibits osteoclastogenesis by functioning as a decoy receptor of ODF, which has recently been identified as the osteoclastogenic factor of osteoblastic origin (15). OPG is highly expressed in bone and liver, liberated into the bloodstream, and thought to function as a circulating factor. The increase in OPG mRNA and serum protein levels in $\mathrm{kl} / \mathrm{kl}$ mice can be interpreted in 2 ways. First, expression of OPG may be under the control of the klotho signaling pathway that controls general aging in vivo. In fact, serum OPG protein level is reported to be significantly increased with age in both healthy men and women (9). Although in vivo upregulation of OPG might cause the impairment of osteoclastogenesis in $\mathrm{kl} / \mathrm{kl}$ mice, osteoclastogenesis in vitro was not reduced when $k l / k l$ osteoblasts expressing higher OPG were cocultured with wild-type hemopoietic cells (Figure 4b). However, this finding does not necessarily deny the possibility that OPG mediates the klotho signal for impaired osteoclastogenesis because the coculture was 
done in the presence of $1 \alpha, 25(\mathrm{OH})_{2} \mathrm{D}_{3}$, which is a potent inducer of ODF and its receptor, RANK (19). Second, upregulation of OPG may be a compensatory mechanism for bone loss in $\mathrm{kl} / \mathrm{kl}$ mice to inhibit further bone resorption. Although serum OPG level is also increased in postmenopausal women with not only low bone mass, but also with a high rate of bone turnover (9), OPG upregulation might not be a cause but a result of bone loss. In any case, increased OPG expression is likely to be involved in the pathophysiology of osteopenia in $\mathrm{kl} / \mathrm{kl}$ mice.

Age-related bone loss in humans differs from postmenopausal bone loss not only with respect to the underlying cellular changes, but also with respect to the skeletal sites affected. The latter occurs primarily in trabecular bone, whereas the former occurs primarily in cortex bone (2). The largest decrease in bone density was observed at the cortex bone of the diaphysis in $k l / k l$ mice, indicating a resemblance to senile osteoporosis. Metaphyses around the knee joint, on the other hand, had elongated trabecular bones with a lamellar architecture and showed high bone density. Mechanism for the focal increase in bone density in this region only remains to be studied further; however, the histomorphometric analysis suggests that this is not a result of focal enhancement of bone formation (Table 1).

Senescence-accelerated mice (SAM) and their substrains are known to exhibit several aging phenotypes, including osteopenia, and have been used as a model for human aging $(20,21)$. Because the conditions of inheritance of the aging phenotypes are very complex in SAM, the genetic foundation has not yet been determined, and multiple gene mutations/polymorphisms are thought to be involved. One of the substrains of SAM, SAMP6, also exhibits low-turnover osteopenia. However, in contrast to $\mathrm{kl} / \mathrm{kl}$ mice, the decreased osteoclastogenesis was shown to be secondary to the osteoblast defect of these mice (22). Thus, the pathogenesis of osteopenia may be different between the 2 mouse models for aging.

The klotho mouse is the first laboratory animal model with multiple phenotypes closely resembling human aging caused by a single gene mutation. Although the aging phenotypes may not be completely identical to the natural aging process in humans, they clearly reflect various aspects of this process. Investigation of the bone abnormality in the klotho mouse is expected to provide new insight into the molecular pathogenesis of senile osteoporosis.

\section{Acknowledgments}

We thank Y. Nabeshima for arrangement of animal production, and C. Pilbeam for discussions. We also thank the hard tissue research team at Kureha Chemical Co. Ltd. for technical assistance. This work was supported by a Grant-in-Aid for Scientific Research from the Japanese Ministry of Education, Science, Sports and Culture (09307033), a grant from Japan Orthopaedics and Traumatology Foundation (0096), and a grant from the Research Society for Metabolic Bone Diseases (all to H. Kawaguchi); and a BristolMyers Squibb/Zimmer Unrestricted Research Grant (to K. Nakamura).

1. Riggs, B.L., and Melton, L.J., III. 1986. Involutional osteoporosis. N. Engl. J. Med. 314:1676-1686.

2. Manolagas, S.C., and Jilka, R.L. 1995. Mechanisms of diseases: bone marrow, cytokines, and bone remodeling - emergency insights into the pathophysiology of osteoporosis. N. Engl. J. Med. 332:305-311.

3. Kuro-o, M., et al. 1997. Mutation of the mouse klotho gene leads to a syndrome resembling aging. Nature. 390:45-51.

4. Shiraki-Iida, T., et al. 1998. Structure of the mouse klotho gene and its two transcripts encoding membrane and secreted protein. FEBS Lett. 424:6-10.

5. Matsumura, Y., et al. 1998. Identification of the human klotho gene and its two transcripts encoding membrane and secreted Klotho protein. Biochem. Biophys. Res. Commun. 242:626-630

6. Yu, C.E., et al. 1996. Positional cloning of the Werner's syndrome gene. Science. 272:258-262.

7. Ellis, N.A., et al. 1995. The Bloom's syndrome gene product is homologous to RecQ helicases. Cell. 83:655-666.

8. Weeda, G., et al. 1990. A presumed DNA helicase encoded by ERCC-3 is involved in the human repair disorders xeroderma pigmentosum and Cockayne's syndrome. Cell. 62:777-791.

9. Yano, K., et al. 1999. Immunological characterization of circulating osteoprotegerin/osteoclastogenesis inhibitory factor: increased serum concentrations in postmenopausal women with osteoporosis. J. Bone Miner. Res. 14:518-527.

10. Weinstein, R.S., Jilka, R.L., Parfitt, A.M., and Manolagas, S.C. 1997. The effects of androgen deficiency on murine bone remodeling and bone mineral density are mediated via cells of the osteoblastic lineage. Endocrinology . 138:4013-4021.

11. Takahashi, N., et al. 1991. Role of colony-stimulating factors in osteoclast development. J. Bone Miner. Res. 6:977-982.

12. Suda, T., Nakamura, I., Jimi, E., and Takahashi, N. 1997. Regulation of osteoclast function. J. Bone Miner. Res. 12:869-879.

13. Komori, T., et al. 1997. Targeted disruption of Cbfa-1 results in a complete lack of bone formation owing to maturational arrest of osteoblasts. Cell. 89:755-764.

14. Yamashita, H., Ten-Dijke, P., and Miyazono, K. 1996. Bone morphogenetic protein receptors. Bone. 19:569-574.

15. Yasuda, H., et al. 1998. Osteoclast differentiation factor is a ligand for osteoprotegerin/osteoclastogenesis-inhibitory factor and is identical to TRANCE/RANKL. Proc. Natl. Acad. Sci. USA. 95:3597-3602.

16. Simonet, W.S., et al. 1997. Osteoprotegerin: a novel secreted protein involved in the regulation of bone density. Cell. 89:309-319.

17. Yasuda, H., et al. 1998. Identity of osteoclastogenesis inhibitory factor (OCIF) and osteoprotegerin (OPG): a mechanism by which OPG/OCIF inhibits osteoclastogenesis in vitro. Endocrinology. 139:1329-1337.

18. Saito, Y., et al. 1998. Klotho protein protects against endothelial dysfunction. Biochem. Biophys. Res. Commun. 248:324-329.

19. Horwood, N.J., Elliott, J., Martin, T.J., and Gillespie, M.T. 1998. Osteotropic agents regulate the expression of osteoclast differentiation factor and osteoprotegerin in osteoblastic stromal cells. Endocrinology. 139:4743-4747.

20. Takeda, T., Hosokawa, M., and Higuchi, K. 1991. Senescence-accelerated mice (SAM): a novel murine model of accelerated senescence. J. Am. Geriatr. Soc. 39:911-919.

21. Matsushita, M., et al. 1986. Age-related changes in bone mass in the senescence-accelerated mouse (SAM). SAM-R/3 and SAM-P/ 6 as new murine models for senile osteoporosis. Am. J. Pathol. 125:276-283.

22. Jilka, R.L., Weinstein, R.S., Takahashi, K., and Manolagas, S.C. 1996. Linkage of decreased bone mass with impaired osteoblastogenesis in a murine model of accelerated senescence. J. Clin. Invest. 97:1732-1740. 